\title{
Checklist of aedine mosquito species (Diptera, Culicidae, Aedini) occurring in Middle and South America (south of the United States) reflecting current generic and subgeneric status
}

\author{
John F. Reinert ${ }^{1}$, Ralph E. Harbach² \& Maria Anice Mureb Sallum ${ }^{3}$
}

${ }^{1}$ Center for Medical, Agricultural and Veterinary Entomology (CMAVE), United States Department of Agriculture, Agricultural Research Service,
1600/1700 SW 23rd Drive, Gainesville, FL 32608-1067 and collaborator, Walter Reed Biosystematics Unit (WRBU), National Museum of
Natural History, Smithsonian Institution, Washington, DC.
${ }^{2}$ Department of Entomology, The Natural History Museum, Cromwell Road, London SW7 5BD, United Kingdom.
${ }^{3}$ School of Public Health, Department of Epidemiology, Medical Entomology Unit for Taxonomic and Systematic Research, São Paulo, Brazil.

ABSTRACT. Checklist of aedine mosquito species (Diptera, Culicidae, Aedini) occurring in Middle and South America (south of the United States) reflecting current generic and subgeneric status. A list of species of tribe Aedini from Middle and South American, south of the United States, with their current generic combinations is provided. Its purpose is to enable entomologists, public health personnel and mosquito control workers to more quickly become familiar with recent formal taxonomic changes within the tribe.

KEYWORDS. Aedini; Culicidae; Checklist.

RESUMO. Lista das espécies de mosquitos aedine (Diptera, Culicidae, Aedini) que ocorrem nas Américas Central e do Sul (ao sul dos Estados Unidos) refletindo o status genérico e subgenérico atual. Apresenta-se lista das espécies da tribo Aedini das Américas Central e do Sul, com a combinação atual de gêneros. Objetiva-se oferecer subsídios aos entomologistas, pessoas dos serviços de saúde e todos os envolvidos com o controle de mosquitos para torna-los familiarizados mais rapidamente com as alterações taxonômicas atuais propostas para a tribo.

PALAVRAS-CHAVE. Aedini; Culicidae; lista das espécies.

Guimarães (1997) listed the species of Culicidae from Middle and South America, south of the United States, and provided a basis for the present checklist of Aedini. Several recent studies resulted in a number of generic and subgeneric changes to the classification of the tribe in this geographic region and other regions of the world. Changes that impact on the Middle and South American fauna included the resurrection of Ochlerotatus Lynch Arribalzaga to generic status from the composite genus Aedes Meigen (Reinert 2000). More recently, Reinert et al. (2004) revised the generic classification of Aedini based on cladistic analyses of all previously recognized genera, subgenera and major species groups of the tribe. As a result of this extensive study, which included 172 morphological characters of all life stages, 46 genera are now recognized for most of the species previously included in 12 genera and 56 subgenera. As required by Articles 31.2 and 34.2 of the International Code of Zoological Nomenclature (INTERNATIONAL COMMISSION ON ZOOLOGICAL NOMENCLATURE 1999), the terminations of many species names were changed to agree in gender with newly recognized genera, but for Middle and South American species this applies only to Stegomyia albopicta (Skuse).

The present paper summarizes the taxonomic changes that apply only to the aedine mosquitoes of Middle and South America, south of the United States. Eight genera and 213 species of Aedini (including 3 nomina dubia of Psorophora Robineau-Desvoidy) occur in this geographic area, but only
120 species are now classified in one or other of the genera. In this geographical area, species previously included in subgenera Ochlerotatus (58 species) and Protomacleaya Theobald (35 species) of Ochlerotatus and one species of subgenus Aedimorphus Theobald of Aedes cannot be placed in a currently recognized genus and are regarded as incertae sedis (a Latin term meaning "of uncertain taxonomic position") within Aedini. These species are indicated in the list below as belonging to genus (subgenus) of authors, e.g., Aedes (Aedimorphus) sensu auctorum. These generic-level designations are not to be interpreted as having formal status. They are merely temporary repositories for the species listed below them and we recommend the generic-level taxa sensu auctorum continue to be used in the accustomed combinations until the generic placement of these species is resolved. By doing so, minimal disruption will occur with the use of species names with their associated generic-level (genus and subgenus) names. As an example, Aedes (Aedimorphus) vexans Meigen is a valid species of uncertain generic and subgeneric placement that was previously placed in subgenus Aedimorphus of Aedes. Once the phylogenetic relationships of incertae sedis species have been analyzed, they will be retained in their current genera, assigned to new genera, or placed in genera resurrected from synonymy with the genera in which they were previously assigned. However, we emphasize that until such studies have been accomplished, incertae sedis species should continue to be cited with their 
accustomed generic and subgeneric affiliations. Studies are currently underway to resolve the taxonomy of the incertae sedis taxa.

In the new classification, genus Aedes is restricted to the former subgenus Aedes. Stegomyia Theobald, formerly a subgenus of Aedes, is now reinstated to its original status as a genus and includes the two imported species St. aegypti (Linnaeus) and St. albopicta. Genus Ochlerotatus is restricted to five species unassigned to subgenus that were previously included in subgenus Ochlerotatus. Four former subgenera of Ochlerotatus, i.e., Howardina Theobald, the monobasic Aztecaedes Zavortink, Gymnometopa Coquillett, and Kompia Aitken, now have full generic status. Haemagogus Williston and Psorophora are still recognized as genera and their subgeneric classifications remain unchanged. Abbreviations used for genera (2 letters) and subgenera (3 letters) of Culicidae follow Reinert (2001) and Reinert et al. (2004). The abbreviations for genera of Aedini in Middle and South America are indicated before the species listed below.

Species with taxonomically valid generic and subgeneric placement

Tribe Aedini Belkin, 1962

Genus Aztecaedes Zavortink, 1972 Az. ramirezi (Vargas \& Downs, 1950)

Genus Gymnometopa Coquillett, 1906 Gy. mediovittatus (Coquillett, 1906)

Genus Haemagogus Williston, 1896

Subgenus Conopostegus Dyar, 1925

Hg. clarki (Galindo, Carpenter \& Trapido, 1952)

Hg. leucocelaenus (Dyar \& Shannon, 1924)

Hg. leucophoebus (Galindo, Carpenter \& Trapido, 1953)

Hg. leucotaeniatus (Komp, 1938)

Subgenus Haemagogus

Hg. acutisentis Arnell, 1973

Hg. aeritinctus Galindo \& Trapido, 1967

Hg. albomaculatus Theobald, 1903

Hg. anastasionis Dyar, 1921

Hg. andinus Osorno-Mesa, 1944

Hg. argyromeris Dyar \& Ludlow, 1921

Hg. baresi Cerqueira, 1960

Hg. boshelli Osorno-Mesa, 1944

Hg. capricornii Lutz, 1904

Hg. celeste Dyar \& Nunez Tovar, 1927

Hg. chalcospilans Dyar, 1921

Hg. chrysochlorus Arnell, 1973

Hg. equinus Theobald, 1903

Hg. iridicolor Dyar, 1921

Hg. janthinomys Dyar, 1921

Hg. lucifer (Howard, Dyar \& Knab, 1913)

Hg. mesodentatus Komp \& Kumm, 1938

Hg. nebulosus Arnell, 1973

Hg. panarchys Dyar, 1921

Hg. regalis Dyar \& Knab, 1906

Hg. soperi Levi-Castillo, 1955
Hg. spegazzinii Brethes, 1912

Hg. splendens Williston, 1896

Hg. tropicalis Cerqueira \& Antunes, 1938

Genus Howardina Theobald, 1903

Hw. albonotatus (Coquillett, 1906)

Hw. allotecnon (Kumm, Komp \& Ruiz, 1940)

Hw. arborealis (Bonne-Wepster \& Bonne, 1920)

Hw. argyrites (Dyar \& Nunez Tovar, 1927)

Hw. aureolineatus (Berlin, 1969)

Hw. aurites Theobald, 1907

Hw. aurivittatus (Cerqueira, 1943)

Hw. bahamensis (Berlin, 1969)

Hw. brevis (Berlin, 1969)

Hw. brevivittatus (Berlin, 1969)

Hw. busckii (Coquillett, 1906)

Hw. cozumelensis (Diaz Najera, 1966)

Hw. ecuadoriensis (Berlin, 1969)

Hw. eleanorae (Berlin, 1969)

Hw. fulvithorax (Lutz, 1904)

Hw. grabhami (Berlin, 1969)

Hw. guatemala (Berlin, 1969)

Hw. guerrero (Berlin, 1969)

Hw. inaequalis Grabham, 1907

Hw. ioliota (Dyar \& Knab, 1913)

Hw. leei (Berlin, 1969)

Hw. lorraineae (Berlin, 1969)

Hw. marinkellei (Berlin, 1969)

Hw. martinezi (Berlin, 1969)

Hw. osornoi (Berlin, 1969)

Hw. pseudodominicii (Komp, 1936)

Hw. quadrivittatus (Coquillett, 1902)

Hw. septemstriatus (Dyar \& Knab, 1907)

Hw. sexlineatus (Theobald, 1901)

Hw. spinosus (Berlin, 1969)

Hw. stenei (Thompson, 1956)

Hw. vanemdeni (Martini, 1931)

Hw. walkeri (Theobald, 1901)

Hw. whitmorei (Dunn, 1918)

Genus Kompia Aitken, 1941

Ko. purpureipes (Aitken, 1941)

Genus Ochlerotatus Lynch Arribalzaga, 1891

Unassigned to subgenus

Oc. dorsalis (Meigen, 1830)

Oc. fulvus (Wiedemann, 1828)

Oc. infirmatus (Dyar \& Knab, 1906)

Oc. scapularis (Rondani, 1848)

Oc. sollicitans (Walker, 1856)

Genus Psorophora Robineau-Desvoidy, 1827

Subgenus Grabhamia Theobald, 1903

Ps. cingulata (Fabricius, 1805)

Ps. columbiae (Dyar \& Knab, 1906)

Ps. confinnis (Lynch Arribalzaga, 1891)

Ps. dimidiata (Cerqueira, 1943)

Ps. discolor (Coquillett, 1903)

Ps. infinis (Dyar \& Knab, 1906)

Ps. insularia (Dyar \& Knab, 1906) 
Ps. jamaicensis Theobald, 1901

Ps. leucocnemis Martini, 1931

Ps. paulli Paterson \& Shannon, 1927

Ps. pruinosa Martini, 1935

Ps.pygmaea (Theobald, 1903)

Ps. santamarinai Broche, 2000

Ps. signipennis (Coquillett, 1904)

Ps. varinervis Edwards, 1922

Subgenus Janthinosoma Lynch Arribalzaga, 1891

Ps. albigenu (Peryassu, 1908)

Ps. albipes (Theobald, 1907)

Ps. amazonica Cerqueira, 1960

Ps. champerico (Dyar \& Knab, 1906)

Ps. circumflava Cerqueira, 1943

Ps. cyanescens (Coquillett, 1902)

Ps. discrucians (Walker, 1856)

Ps. ferox (von Humboldt, 1819)

Ps. fiebrigi Edwards, 1922

Ps. forceps Cerqueira, 1939

Ps.johnstonii (Grabham, 1905)

Ps. lanei Shannon \& Cerqueira, 1943

Ps. lutzii (Theobald, 1901)

Ps. melanota Cerqueira, 1943

Ps. mexicana (Bellardi, 1859)

Ps. pilosus Duret, 1971

Ps. pseudoalbipes Duret, 1971

Ps. pseudomelanota Barata \& Cotrim, 1971

Ps. totonaci Lassmann, 1951

Ps. varipes (Coquillett, 1904)

Subgenus Psorophora

Ps. ciliata (Fabricius, 1794)

Ps. cilipes (Fabricius, 1805)

Ps. holmbergii Lynch Arribalzaga, 1891

Ps. howardii Coquillett, 1901

Ps. lineata (von Humboldt, 1819)

Ps. ochripes (Macquart, 1850)

Ps. pallescens Edwards, 1922

Ps. pilipes (Macquart, 1834)

Ps. saeva Dyar \& Knab, 1906

Ps. stonei Vargas, 1956

Nomina dubia

Ps. goeldii (Giles, 1904)

Ps. marmorata (Phillipi, 1865)

Ps. perterrens (Walker, 1856)

Genus Stegomyia Theobald, 1901

St. aegypti (Linnaeus, 1762)

St. albopicta (Skuse, 1895)

Incertae sedis species with provisional generic and subgeneric placement

Genus Aedes Meigen, 1818, sensu auctorum

Subgenus Aedimorphus Theobald, 1903, sensu auctorum

Ae. vexans vexans (Meigen, 1830)

Genus Ochlerotatus Lynch Arribalzaga, 1891, sensu auctorum

Subgenus Ochlerotatus Lynch Arribalzaga, 1891, sensu auctorum
Oc. aenigmaticus (Cerqueira \& Costa, 1946)

Oc. albifasciatus (Macquart, 1838)

Oc. angustivittatus (Dyar \& Knab, 1907)

Oc. annuliventris (Blanchard, 1852)

Oc. atactavittatus (Arnell, 1976)

Oc. auratus (Grabham, 1906)

Oc. bejaranoi (Martinez, Carcavallo \& Prosen, 1960)

Oc. bimaculatus (Coquillett, 1902)

Oc. bogotanus (Arnell, 1976)

Oc. calumnior (Belkin, Heinemann \& Page, 1970)

Oc. campestris (Dyar and Knab, 1907)

Oc. canadensis (Theobald, 1901)

Oc. comitatus (Arnell, 1976)

Oc. condolescens (Dyar \& Knab, 1907)

Oc. crinifer (Theobald, 1903)

Oc. deficiens (Arnell, 1976)

Oc. dupreei (Coquillett, 1904)

Oc. epactius (Dyar \& Knab, 1908)

Oc. eucephalaeus (Dyar, 1918)

Oc. euiris (Dyar, 1922)

Oc. euplocamus (Dyar \& Knab, 1906)

Oc. fluviatilis (Lutz, 1904)

Oc. hastatus (Dyar, 1922)

Oc. hortator (Dyar \& Knab, 1907)

Oc. incomptus (Arnell, 1976)

Oc. jacobinae (Serafim \& Davis, 1933)

Oc. laguna (Arnell \& Nielsen, 1972)

Oc. lepidus (Cerqueira \& Paraense, 1945)

Oc. martineti (Senevet, 1937)

Oc. meprai (Martinez \& Prosen, 1953)

Oc. milleri (Dyar, 1922)

Oc. mitchellae (Dyar, 1905)

Oc. monticola (Belkin \& McDonald, 1957)

Oc. muelleri (Dyar, 1920)

Oc. nubilus (Theobald, 1903)

Oc. obturbator (Dyar \& Knab, 1907)

Oc. oligopistus (Dyar, 1918)

Oc. patersoni (Shannon \& del Ponte, 1928)

Oc. pectinatus (Arnell, 1976)

Oc. pennai (Antunes \& Lane, 1938)

Oc. pertinax (Grabham, 1906)

Oc. perventor (Cerqueira \& Costa, 1946)

Oc. phaeonotus (Arnell, 1976)

Oc. raymondi (del Ponte, Castro \& Garcia, 1951)

Oc. rhyacophilus (da Costa Lima, 1933)

Oc. scutellalbum (Boshell-Manrique, 1939)

Oc. serratus (Theobald, 1901)

Oc. shannoni (Vargas \& Downs, 1950)

Oc. spencerii (Theobald, 1901)

Oc. squamiger (Coquillett, 1902)

Oc. stigmaticus (Edwards, 1922)

Oc. synchitus (Arnell, 1976)

Oc. taeniorhynchus (Wiedemann, 1821)

Oc. thelcter (Dyar, 1918)

Oc. tormentor (Dyar \& Knab, 1906)

Oc. tortilis (Theobald, 1903)

Oc. trivittatus (Coquillett, 1902) 
Oc. upatensis (Anduze \& Hecht, 1943)

Subgenus Protomacleaya Theobald, 1907, sensu auctorum

Oc. aitkeni (Schick, 1970)

Oc. alboapicus (Schick, 1970)

Oc. argyrothorax (Bonne-Wepster \& Bonne, 1920)

Oc. berlini (Schick, 1970)

Oc. bertrami (Schick, 1970)

Oc. braziliensis (Gordon \& Evans, 1922)

Oc. buenaventura (Schick, 1970)

Oc. burgeri (Zavortink, 1972)

Oc. campana (Schick, 1970)

Oc. casali (Schick, 1970)

Oc. chionotum (Zavortink, 1972)

Oc. daryi (Schick, 1970)

Oc. diazi (Schick, 1970)

Oc. gabriel (Schick, 1970)

Oc. galindoi (Schick, 1970)

Oc. heteropus (Dyar, 1921)

Oc. homoeopus (Dyar, 1922)

Oc. idanus (Schick, 1970)

Oc. impostor (Schick, 1970)

Oc. insolitus (Coquillett, 1906)

Oc. knabi (Coquillett, 1905)

Oc. kompi (Vargas \& Downs, 1950)

Oc. metoecopus (Dyar, 1925)

Oc. niveoscutum (Zavortink, 1972)

Oc. podographicus (Dyar \& Knab, 1906)

Oc. sandrae (Zavortink, 1972)

Oc. schicki (Zavortink, 1972)

Oc. schroederi (Schick, 1970)

Oc. sumidero (Schick, 1970)

Oc. tehuantepec (Schick, 1970)
Oc. terrens (Walker, 1856)

Oc. thorntoni (Dyar \& Knab, 1907)

Oc. vargasi (Schick, 1970)

Oc. zavortinki (Schick, 1970)

Oc. zoosophus (Dyar and Knab, 1918)

Acknowledgements. Appreciation is expressed to Kenneth $\mathrm{J}$ Linthicum (CMAVE) for providing facilities to JFR. Special thanks are extended to Bruce A. Harrison, (Public Health Pest Management, North Carolina Department of Environment and Natural Resources, Winston-Salem, NC) and Graham B. White (Department of Entomology, University of Florida, Gainesville, FL) for reviewing the manuscript.

\section{REFERENCES}

Guimarães, J. H. 1997. Systematic Database of Diptera of the Americas South of the United States (Family Culicidae). Fundação de Amparo a Pesquisa do Estado de São Paulo. São Paulo, Brazil, ix + 286 p.

INTERNATIONAL COMMISSION ON ZOOLOGICAL NOMENCLATURE. 1999. International Code of Zoological Nomenclature. Fourth Edition. International Trust for Zoological Nomenclature. London, United Kingdom, xxviii + 306 p.

Reinert, J. F. 2000. New classification for the composite genus Aedes (Diptera: Culicidae: Aedini), elevation of subgenus Ochlerotatus to generic rank, reclassification of the other subgenera, and notes on certain subgenera and species. Journal of the American Mosquito Control Association 16: 75-188.

Reinert, J. F. 2001. Revised list of abbreviations for genera and subgenera of Culicidae (Diptera) and notes on generic and subgeneric changes. Journal of the American Mosquito Control Association 17: $51-55$.

Reinert J. F., R. E. Harbach \& I. J. Kitching. 2004. Phylogeny and classification of Aedini (Diptera: Culicidae) based on morphological characters of all life stages. Zoological Journal of the Linnean Society 142: 289-368. 